\title{
Spinal cord injury secondary to cervical disc herniation in ambulatory patients with cerebral palsy
}

\author{
Hyun-Yoon $\mathrm{Ko}^{1}$ and Insun Park-Ko ${ }^{2}$ \\ Department of Rehabilitation Medicine, ${ }^{1}$ Pusan National University Hospital, Pusan National University College of \\ Medicine, and ${ }^{2}$ Inje University Pusan Paik Hospital, Pusan, Korea
}

\begin{abstract}
Early onset of degeneration of the cervical spine and instability due to sustained abnormal tonicity or abnormal movement of the neck are found in patients with cerebral palsy. An unexplained change or deterioration of neurological function in patients with cerebral palsy should merit the consideration of the possibility of cervical myelopathy due to early degeneration or instability of the cervical spine. We describe two patients who had a spinal cord injury due to a cervical disc herniation, one patient was athetoid and the second had spastic diplegia, they both had cerebral palsy. It is not easy to determine whether new neurological symptoms are as a result of the cervical spinal cord disorder. These cases suggest that consideration of a cervical spine disorder with myelopathy is required in the evaluation of patients with cerebral palsy who develop deterioration of neurological function or activities over a short period of time.
\end{abstract}

Keywords: spinal cord injury; cerebral palsy; cervical disc herniation

\section{Introduction}

There is a small, but growing body of literature on the later-life complications of congenital or early-onset acquired disabilities. ${ }^{1}$ Aging in patients with cerebral palsy amongst those with neuromuscular disorders has been extensively studied. This study of the aging process and complications serves as a prototype for the assessment of patients with neuromuscular disorders.

Prolonged abnormal tonicity or posture is a well known cause of structural abnormalities in the spine with or without myelopathy In patients with longstanding or congenital abnormal movements, spondylosis and subsequent myelopathy may occur earlier and with a greater frequency compared to the effects of aging in the nondisabled population. ${ }^{2}$ Review of the literature, however, showed that secondary myelopathy in cerebral palsy patients has only been described in case reports. To our knowledge, there is no human study to clarify the relationship between early degeneration of the spine and abnormal tonicity or abnormal movements.

In 1962, Anderson et al. ${ }^{3}$ provided the first report on cervical myelopathy associated with athetoid cerebral palsy. The report described that athetoid neck movements may cause excessive axial neck rotations as well as flexion and extension movements of the spine. Sustained extension tonicity of the neck in those with spastic cerebral palsy may produce an

Correspondence: Hyun-Yoon Ko, M.D., Department of Rehabilitation Medicine, Pusan National University Hospital, 1-10 AmiDong, Suh-Ku, Pusan 602-739, Korea excessive compressive load on the dorsal aspect of the disc. The extended duration of this shearing force or loading in the spine exerts an early degeneration of the corresponding cervical spine, with or without myelopathy.

We describe two patients who have a spinal cord damage caused by a cervical disc herniation in an athetoid and in a spastic diplegic cerebral palsied patient in this report. We also wish to emphasize recent worsening of the neurological abnormality due to cervical spinal cord involvement secondary to early degeneration of cervical spine in patients with cerebral palsy.

\section{Case reports}

Case 1

A 39-year-old male patient with athetoid cerebral palsy complained of pain in both shoulders and progressive weakness of all of his extremities. Over a period of 1 month, he gradually lost his strength of the hands and legs, his ability to walk unaided, and to write. Before this illness, he was able to walk independently, as a community walker defined as a person with the ability to walk indoors and outdoors with or without an orthosis or a walking aid.

Examination on admission showed increased tone, bilateral ankle clonus and Babinski signs in the lower extremities. He had decreased sensation to pinprick below both $\mathrm{C} 5$ dermatomes. There was muscle atrophy 
of both deltoid and biceps muscles with $3 / 5$ motor strength. Both biceps reflexes were absent. He was unable to dress himself independently and was confined to his wheelchair. Athetoid movement of the head and neck were observed. He said that the involuntary movement had decreased with this illness. Urinary bladder function was normal.

Plain films of the cervical spine showed mild kypholordosis and mild anterior listhesis of $\mathrm{C} 3$ on C4 with spondylosis (Figure 1a). An axial CT scan

a

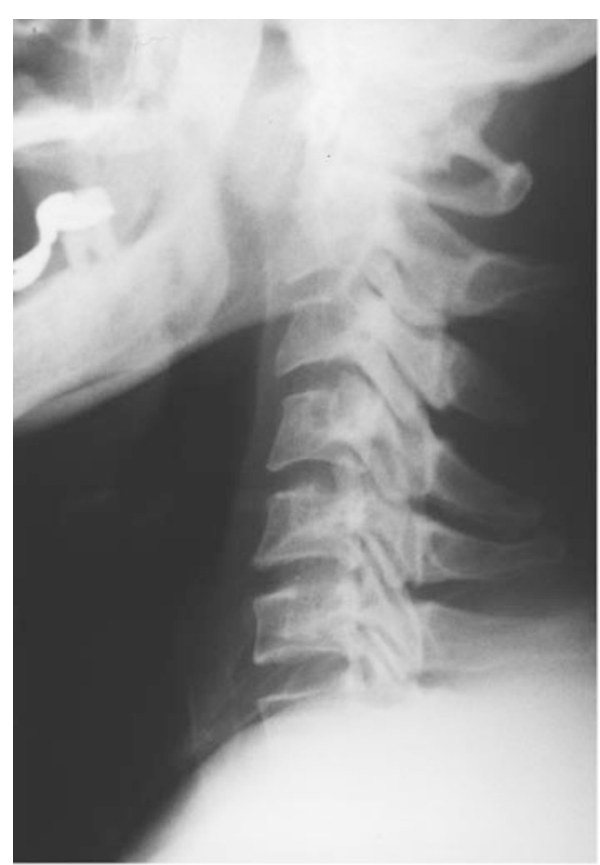

C

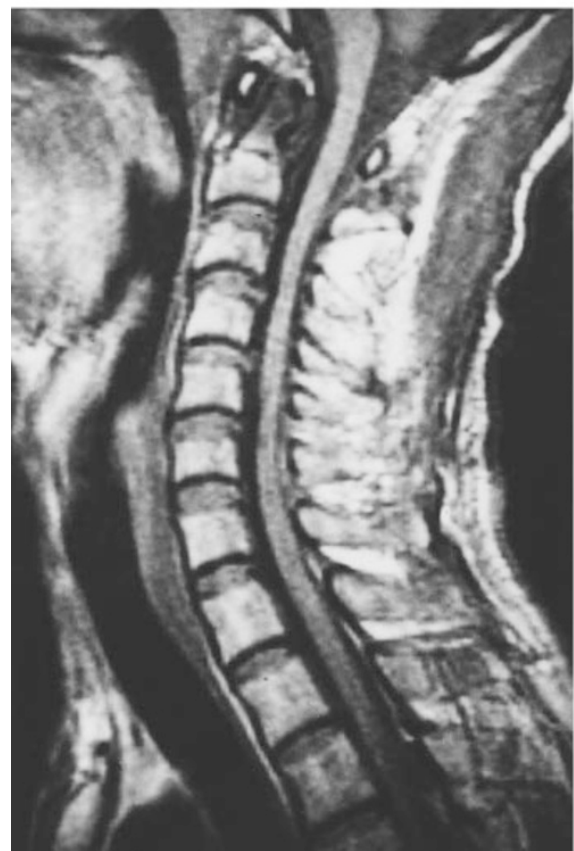

b
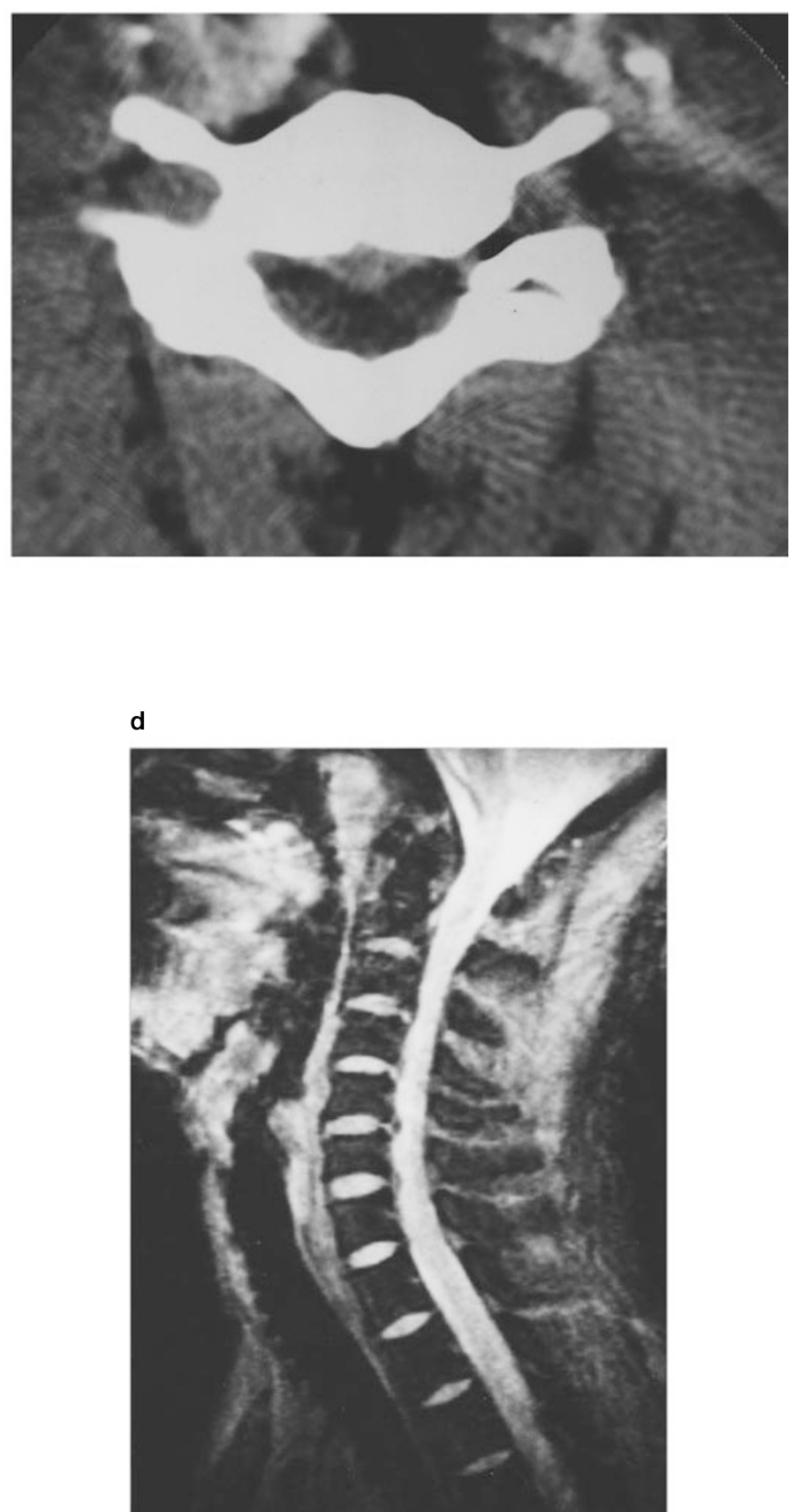

Figure 1 Radiographic images in Case 1. (a) Lateral view of the cervical spine showing kypholordosis and mild anterior listhesis of C3 on C4. (b) Axial CT showing central disc protrusion at C5-C6. (c) T1-weighted MRI showing low intensity signals of the anterior portion of the spinal cord at C5 and C6. (d) T2-weighted MRI showing herniated disc at C3-C4 to C6C7 
without contrast at a level $(\mathrm{C} 5-\mathrm{C} 6)$ of the cervical spine revealed a central protrusion of the disc (Figure 1b). The sagittal T1-weighted MR image defined an area of low signal intensity within the anterior portion

\section{a}

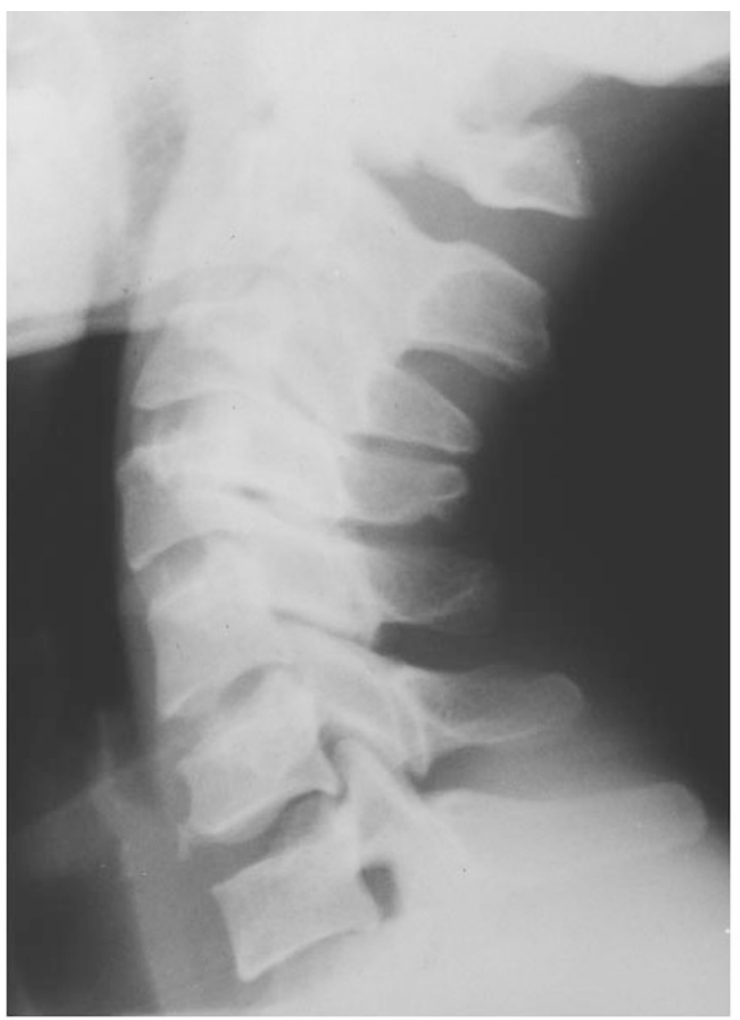

of the spinal cord at C5-C6 (Figure 1c). Midline sagittal T2-weighted MR image of the cervical spine showed a narrowed canal with herniated disc, impinging on the spinal cord at $\mathrm{C} 3-\mathrm{C} 4$ to $\mathrm{C} 6-\mathrm{C} 7$ b

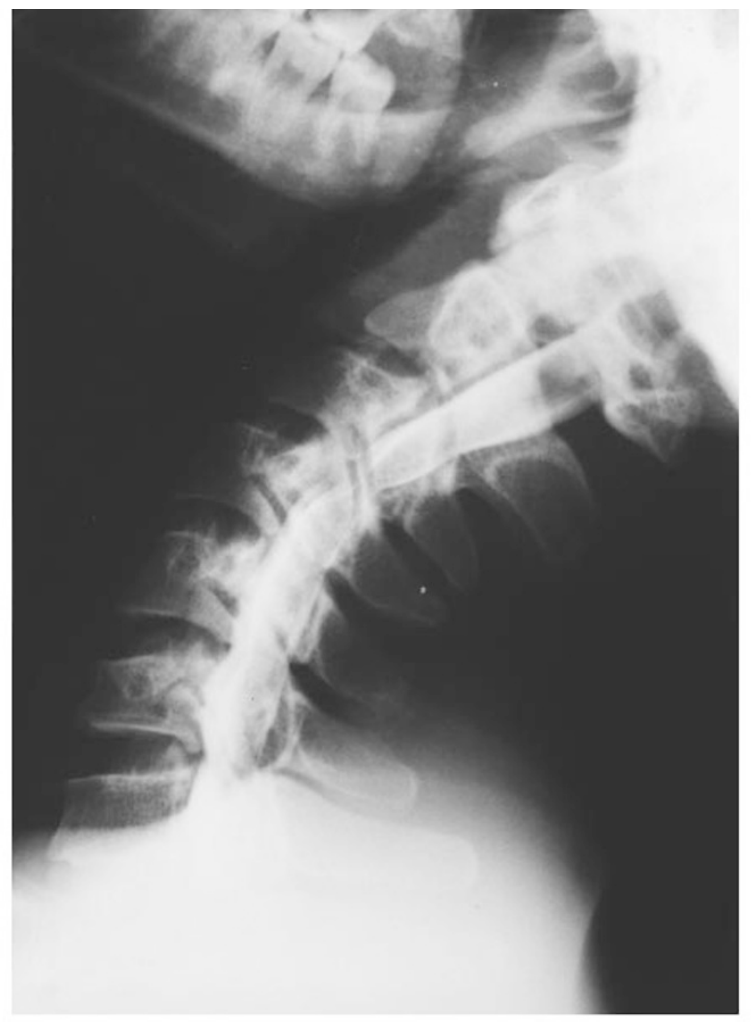

c

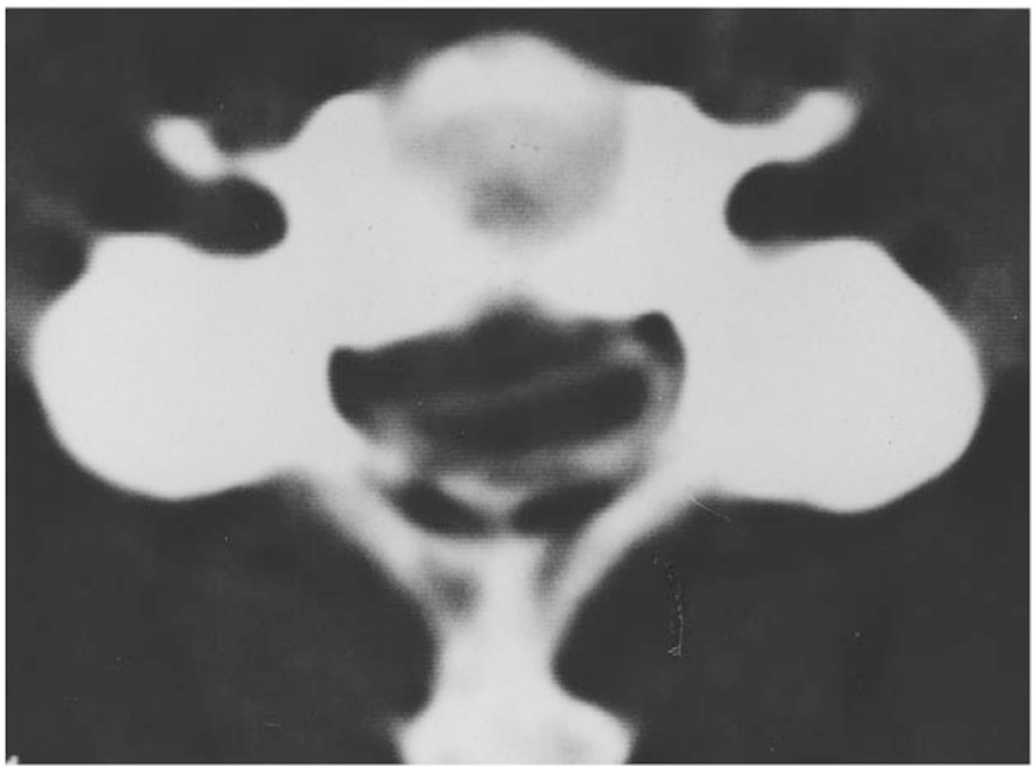

Figure 2 Radiographic images in Case 2. (a) Lateral view of the cervical spine showing increased lordosis. (b) Lateral myelography showing a partial block at $\mathrm{C} 3-\mathrm{C} 4$ with puckering of the ligamentum flavum. (c) $\mathrm{CT}$-myelography at $\mathrm{C} 3-\mathrm{C} 4$ showing intervertebral disc protrusion 
(Figure 1d). There was no benefit from treatment with nonsteroid antiinflammatory drugs and cervical immobilization using a Philadelphia collar. He underwent an anterior decompression and interbody fusion of $\mathrm{C} 5-\mathrm{C} 6$ and $\mathrm{C} 6-\mathrm{C} 7$. After surgery, the athetoid movement in his extremities reappeared gradually and his strength increased. At follow-up examination two months later, he was able to ambulate with the minimal assistance of one person. The Barthel Index Score was improved to 70 from 35 . One year later, he regained the ability to walk with no walking aid.

\section{Case 2}

A 28-year-old male patient with spastic diplegic cerebral palsy developed pain and weakness in his right extremities over a 1 month-period. The patient reported that his gait was worsened gradually with this illness. Previously, he had been able to walk independently on his own without an assistive device. Physical examination revealed symmetrical hyperreactive deep tendon reflexes in the upper and lower extremities. There was ankle clonus and Babinski signs. $\mathrm{He}$ had diminished pinprick sensation below C5 dermatomes, bilaterally. Motor weakness of the legs was worse than his arms and his right side was worse than his left side. The neurological deterioration was gradually becoming more severe. The cranial nerves were normal. Urinary bladder function was normal. The Barthel Index Score was 75. When he was admitted, neurological evaluations were performed on a possible brain new problem. However, a brain CT and an EEG showed no abnormality. Because the neurological deterioration was continued, further evaluation was given on cervical spine.

Cervical spine radiograph showed degenerative changes and increased lordosis in the cervical spine (Figure 2a). A cervical myelogram revealed a partial block and acute angulation at $\mathrm{C} 3-\mathrm{C} 4$ with compression by a protruded disc ventrally and compression dorsally by the puckering of the ligamentum flavum with neck extension (Figure 2b). A Metrizamideenhanced $\mathrm{CT}$ of the cervical spine showed a central and posterolateral protrusion of the $\mathrm{C} 3-\mathrm{C} 4$ intervertebral disc (Figure 2c). An anterior cervical discectomy and an interbody fusion were performed on the patient. He gradually improved postoperatively. Two months later, he was able to ambulate with a cane in his left hand. His Barthel Index Score at discharge improved to 100 .

\section{Discussion}

In evaluating late neurological and functional deterioration in patients with cerebral palsy, several factors should be considered: a new brain lesion, secondary disuse or misuse complications in the extremities, a spinal and/or spinal cord disorder related to the long duration of abnormal movements or tonicity. In the patient with cerebral palsy. mild changes in tone, strength, and other neurological signs may not be clearly defined. Even if recognized in a patient with a preexisting congenital disability, such neurological changes do not often give rise to medical concern until they become severe. If such patients are mentally retarded, early recognition of the change can be delayed even longer.

Cervical radiculopathy or myelopathy resulting from spondylosis in the nondisabled population usually appears in the sixth or seventh decade. ${ }^{4}$ In patients with involuntary movements or/and abnormal tonicity, spondylotic changes in the cervical spine will occur at a younger age than happens in the nondisabled population. ${ }^{5}$ Spondylotic changes can be seen within 10 years of the development of dyskinesia, but signs of myelopathy usually develop later. ${ }^{2}$ This premature onset of spondylosis appears to be related to the characteristic rapid and repetitious neck movements. ${ }^{6,7}$

Several authors have reported that patients with cerebral palsy develop early degenerative changes of the cervical spine and radiculomyelopathy resulting from excessive rotation, flexion and extension of the cervical spine. . $3,5,8-14$ However, there is no human study to clarify the relationship between early degeneration of the spine and abnormal tonicity or abnormal movement.

A larger range of motion of the cervical spine of patients with cerebral palsy suggests the absence of a deceleration mechanism at the terminal phase of the extension-flexion motion. ${ }^{6}$ The lack of a smooth gradient for velocity and acceleration according to the level of the vertebra, or a sudden change in velocity during extension-flexion motion results in a malfunction in the control mechanism of cervical motion. ${ }^{6}$ The bending movement and shearing force that work on the cervical spine of patients with athetoid cerebral palsy is greater than that which occurs in normal controls. ${ }^{6}$

To our knowledge, an animal study by Wada et al. ${ }^{15}$ in 1992 is the only in vivo study showing that overuse from repetitive movement can accelerate the progression of cervical spondylosis. Their results demonstrated that senescence is not the only factor in the development of cervical spondylosis.

The C5-C6 interspace in the nondisabled population is the most common site of segmental instability because this is the site of maximum flexion and extension. ${ }^{16}$ In comparison, multiple segmental instability of the cervical spine is common in those with athetoid cerebral palsy. ${ }^{16}$ Segmental instability is often situated at $\mathrm{C} 3-\mathrm{C} 4$ and $\mathrm{C} 4-\mathrm{C} 5$, and occasionally at C5-C6 in those with athetoid cerebral palsy. ${ }^{16}$ To date, most reports describing neurological deterioration secondary to early degeneration of cervical spine in people with cerebral palsy were on those with athetoid cerebral palsy. Reese et al. ${ }^{5}$ described the only patients with neurological deterioration in individuals with spastic cerebral palsy resulting from cervical spine pathology. 
In disabled persons with recent neurological deterioration, the previous level of independence or functional skill as well as recent neurological status must be assessed and documented. Diagnosis and localization of the responsible lesion can not be easily defined since neurological deficits are complicated and overloaded by cervical myelopathy, which further worsens existing cerebral motor dysfunctions in cerebral palsied patients. We present these patients in our report to increase awareness of the possibility of cervical spine pathology in these cerebral palsied adults with long duration of abnormal tonicity or involuntary movements.

\section{References}

1 Currie DM, Gershkoff AM, Cifu AX. Geriatric rehabilitation. 3. Mid- and late-life effects of early-life disabilities. Arch Phys Med Rehabil 1993; 74: S413-416.

2 El-Mallakh RS, Rao K, Barwick M. Cervical myelopathy secondary to movement disorders: case report. Neurosurg 1989; 4: $902-905$.

3 Anderson WW, Wise BL, Itabashi HH, Jones M. Cerebral spondylosis in patients with athetosis. Neurology 1962; 12: 410412.

4 Clarke E, Robinson PK. Cervical myelopathy: a complication of cervical spondylosis. Brain 1956; 79: 483-510.

5 Reese ME et al. Acquired cervical spine impairment in young adults with cerebral palsy. Dev Med Child Neurol 1991; 33: $153-$ 166.
6 Ebara S et al. Motion analysis of the cervical spine in athetoid cerebral palsy: extension-flexion motion. Spine 1990; 15: $1097-$ 1103.

7 Ebara S et al. Unstable cervical spine in athetoid cerebral palsy. Spine 1989; 14: 1154-1159.

8 Fusi T et al. Cervical radiculopathy or myelopathy secondary to athetoid cerebral palsy. J Bone Joint Surg 1987; 69-A: 815-821.

9 Hanakita $\mathbf{J}$ et al Surgical treatment of cervical spondylotic radiculomyelopathy with abnormal involuntary neck movements. Neurol Med Chir (Tokyo) 1989; 29: 1132-1136.

10 Nishihara $\mathrm{N}$ et al. Surgical treatment of cervical spondylotic myelopathy complicating athetoid cerebral palsy. J Bone Joint Surg (Am) 1984; 66-B: $504-508$.

11 Hirose G, Kadoya S. Cervical spondylotic radiculo-myelopathy in patients with athetoid-dystonic cerebral palsy: clinical evaluation and surgical treatment. J Neurol Neurosurg Psychiatry $1984 ;$ 47: $775-880$.

12 Kidron D, Steiner I, Melamed E. Late-onset progressive radiculomyelopathy in patients with cervical athetoid-dystonic cerebral palsy. Eur Neurol 1987; 27: 164-166.

13 Rosenberg NJ, Bargar WL, Friedman B. The incidence of spondylolysis and spondylolisthesis in nonambulatory patients. Spine 1981; 6: $35-38$.

14 McCluer S. Cervical spondylosis with myelopathy as a complication of cerebral palsy. Paraplegia 1982; 20: $308-312$.

15 Wada E, Ebara S, Saito S, Ono K. Experimental spondylosis in the rabbit spine: overuse could accelerate the spondylosis. Spine 1992; 35: S1-6.

16 Odom GL, Finney W, Woodhall B. Cervical disc lesions. J Am Med Ass 1958; 166: $23-28$ 